\title{
Evaluation parameters of graft maturation on second-look arthroscopy following anterior cruciate ligament reconstruction: a systematic review
}

\author{
Sang-Gyun Kim¹, Jae Hyun Jung², Jong-Hyub Song ${ }^{1}$ and Ji-Hoon Bae ${ }^{1 *}$ (D)
}

\begin{abstract}
Purpose: The purpose of this systematic review was to investigate and summarize the evaluation methods of graft maturation on second-look arthroscopy following anterior cruciate ligament $(\mathrm{ACL})$ reconstruction.

Methods: A literature search was performed on articles before December 2017 to identify the literature that has evaluated graft maturation on second-look arthroscopy following ACL reconstruction. Only studies using human grafts, evaluating graft maturation with two or more gross findings were included. Study design, grafts, surgical techniques, follow-up period, evaluation parameters, and categories were compiled.
\end{abstract}

Results: Twenty-eight studies were included in this study. All studies evaluated graft maturation with two or more of the following three findings: graft integrity, tension, and synovial coverage. Two to four categories were used for evaluating each parameter, but the criteria for classification were slightly different for each study. Several studies reported neo-vascularization of grafts and the total maturation score by summing up the scores assigned to each evaluation parameter. Three studies reported that there was no correlation between second-look findings and patient-reported outcomes.

Conclusions: Graft integrity, tension, and synovial coverage were the most frequently evaluated for graft maturation on second-look arthroscopy. However, there is no uniform criterion for evaluation. Therefore, development of a valid, uinform criterion is required.

Level of evidence: Level IV, systematic review of level I-IV investigations.

Keywords: Knee, Anterior cruciate ligament reconstruction, Second-look surgery, Graft, Maturation, Systematic review

\section{Introduction}

After anterior cruciate ligament (ACL) reconstruction, the graft tendon undergoes a maturation process called "ligamentization". Amiel et al. [1] described this phenomenon as the continuous development of a tissue, which was a tendon originally, into a substance very similar to a normal ACL. For long-term survival of grafts, favorable biologic quality with good ligamentization as well as mechanical properties would be essential $[2,3]$.

\footnotetext{
* Correspondence: osman@korea.ac.kr

${ }^{1}$ Department of Orthopaedic Surgery, Korea University Guro Hospital, Korea University College of Medicine, 148 Gurodong-ro, Guro-gu, Seoul 08308, Republic of Korea

Full list of author information is available at the end of the article
}

Recent studies have shown that autograft is superior to allograft in long-term survival $[2,4]$, which may be different in biologic maturation.

Many studies have evaluated graft maturation using second-look arthroscopy [5-9]. This method has several disadvantages. Histologic findings cannot be confirmed and the maturation of the graft depends on the subjective evaluation of the surgeon. However, second-look arthroscopy has several advantages over biopsy. It does not cause damage to the grafts by evaluating the graft maturation indirectly. Also, it doesn't result in different outcomes depending on the site from where the tissue is taken. For these reasons, many studies have evaluated graft 
maturation with gross findings seen in the secondlook arthroscopy until recently [10-14].

However, there was no consensus on which parameters should be evaluated and which criteria should be used for each parameter to evaluate graft maturation on secondlook arthroscopy. Surgeons have been using their own criteria for evaluating graft maturation. Toritsuka et al. [5] evaluated graft maturation by classifying the integrity and tension of the graft observed on the second-look arthroscopy into four categories, whereas Ahn et al. [6] and Kondo et al. [7] evaluated it by classifying the integrity, tension, and synovial coverage of grafts into three categories.

The purpose of this study was to review the previous literature (1) to investigate how to evaluate graft maturation on second-look arthroscopy following ACL reconstruction and (2) to determine if second-look arthroscopy had an objective evaluation value. We hypothesized there would be a lot of different methods to perform second-look arthroscopy following ACL reconstruction and second-look findings would be less correlated to the clinical outcomes.

\section{Methods}

\section{Study eligibility criteria}

We performed a systematic review of all the literature on second-look arthroscopy following ACL reconstruction, regardless of the level of study, graft type, and surgical technique (femoral tunnel placement, single bundle or double bundle reconstruction). Searches were restricted to papers published in English. Only articles with detailed description on how to evaluate graft maturation were included. The mean follow-up period of all included studies was more than one year.

\section{Literature search}

Our literature search consisted of searches in PubMed and Embase for the terms "anterior cruciate ligament", "reconstruction", and "second-look" from the inception of these search engines till December 2017. After removing duplications, we checked the titles and abstracts of all articles to determine whether they fit the previously established inclusion criteria. The included articles identified by the search were each analyzed by a senior author to ensure their appropriateness.

Only human ACL reconstruction studies using human grafts and evaluating graft maturation on second-look arthroscopy with two or more gross findings were included. As it was difficult to comprehensively evaluate graft maturation with only one parameter, we included only those studies that evaluated two or more gross findings of the grafts. In addition, studies with synthetic grafts, case reports, studies for which only abstracts had been published without full text, and systematic review studies were excluded. The results of this literature review are outlined in the Preferred Reporting Items for Systematic Reviews and Meta-Analyses (PRISMA) diagram in Fig. 1.

\section{Data extraction}

The data from each of the 28 articles meeting the inclusion criteria for our systematic review were compiled. We collected demographic data, such as the graft type, number of second-look arthroscopies performed, and follow-up period from reconstruction to second-look arthroscopy. The reconstruction techniques (surgical techniques for femoral tunnel placement, single bundle or double bundle reconstruction) were recorded from all studies that reported them. We investigated the parameters used for evaluating second-look arthroscopy and categories that were used for each parameter to evaluate graft maturation in those studies.

\section{Study quality assessment}

To assess the methodological quality, the modified Coleman Methodology Scores (mCMS) and subscales were determined for each included study [15]. The final score ranges from 0 to 100 , with a score of 100 indicating the highest study quality. In addition, all included studies were assessed for level of evidence according to the Oxford Centre for Evidence-Based Medicine [16]. Most studies had retrospective, non-randomized designs. There were only five randomized controlled trials or prospective cohort studies (levels of evidence I and II) $[7,8,14,17,18]$, probably because of the invasive nature of second-look arthroscopy. Eleven of the remaining 23 studies were retrospective comparative studies (level of evidence III) and 12 studies were case series (level of evidence IV).

\section{Results}

An overview of the 28 studies showing year and journal of publication, level of evidence, graft type, number of second-look arthroscopies performed, reconstruction technique used, and follow-up period from ACL reconstruction to second-look arthroscopy is provided in Table 1. The parameters and categories for evaluating graft maturation on second-look arthroscopy are summarized in Table 2.

\section{Study identification and characteristics}

The literature review described above yielded 28 manuscripts that met all inclusion criteria (see PRISMA flow diagram in Fig. 1). All studies except one [19] were from northeast Asian regions including South Korea [6, 9-11, $14,20,22,24,26,27,31,33]$, Japan [5, 7, 8, 12, 13, 17, 21, $23,25,28-30,32]$, and China [18, 34]. Year of publication ranged from 1994 to 2017. Various grafts (autograft, allograft) were used and the mean follow-up period from 


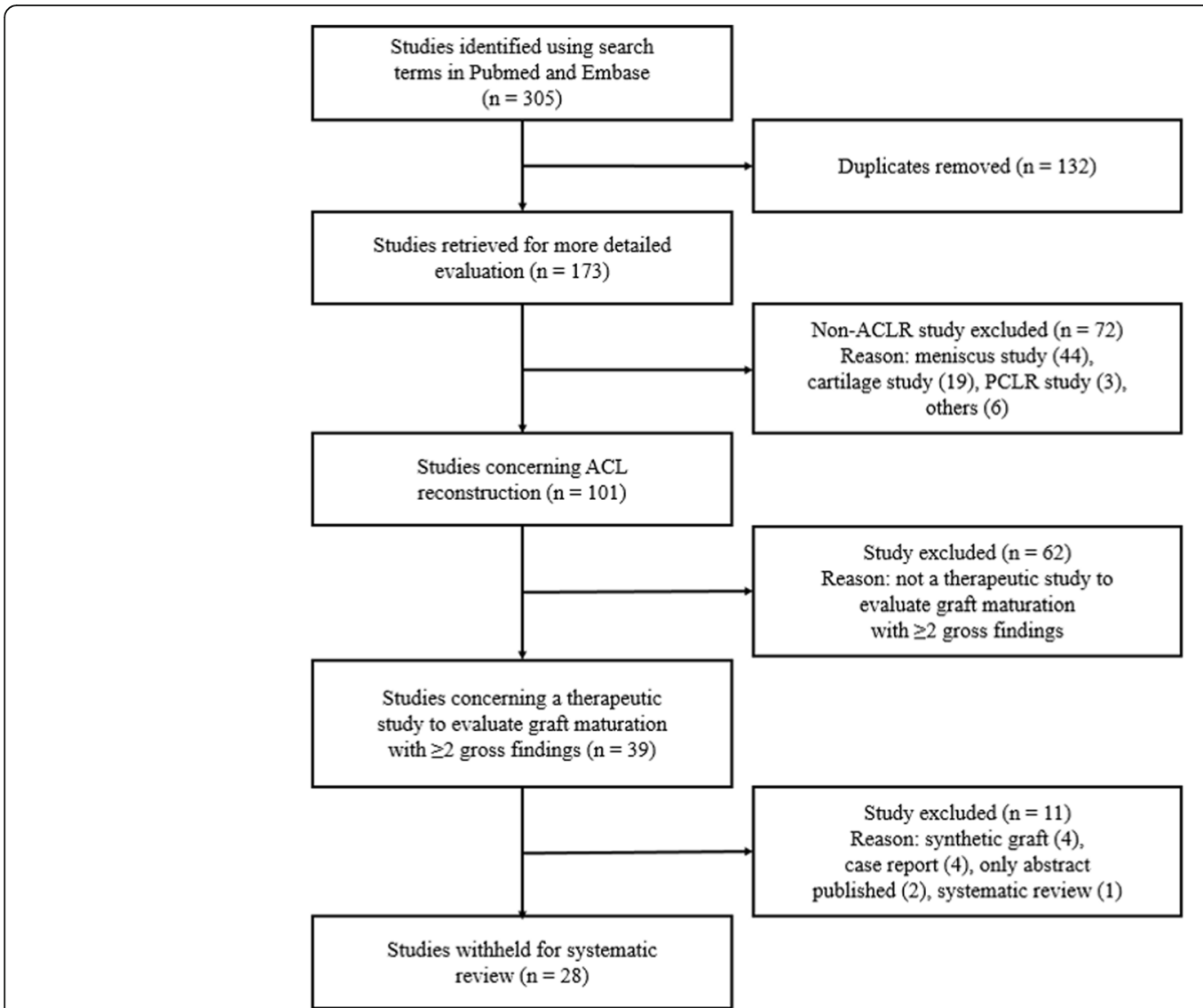

Fig. 1 Quality of Reporting of Meta-analyses (QUOROM) flow diagram depicting the number of studies identified, included, and excluded as well as the reasons for exclusion

reconstruction to second-look arthroscopy was more than 1 year. Regarding reconstruction technique, anatomical and non-anatomical reconstructions and single bundle and double bundle reconstructions were all included (there was one triple bundle reconstruction by Tanaka et al. [30]). All studies evaluated graft maturation with two or more of the following three gross findings: graft integrity, tension, and synovial coverage. Most of the studies had evaluated each parameter separately, while some papers evaluated two or more parameters together $[7,19,20]$. The mean mCMS was $46.4 \pm 6.7$ (range 39 to 61). The corresponding values for each study are shown in Table 1.

\section{Graft integrity on second-look arthroscopy}

Most studies (27/28) evaluated the integrity of the graft on second-look arthroscopy. The most common classification of graft integrity involved three categories according to the severity of graft tear: intact, partial (or superficial) tear, and complete (or substantial) tear [13, 25, 31]. Moreover, five studies categorized the percentage of the intact portion of the entire graft volume [9, 10, 18, 28, 29]. Ohsawa et al. $[28,29]$ described the integrity of the graft as "no tear" when more than $80 \%$ of the graft was intact, "partial tear" when $30-80 \%$ of the graft was intact, and "complete tear" when less than $30 \%$ of the graft was intact. Subsequently, other studies also used these criteria to assess the integrity of the graft $[10,18]$. On the other hand, Ahn et al. [9] classified graft integrity in four categories according to their own criteria ( $\geq 90 \%, 75-90 \%$, $50-75 \%,<50 \%)$.

\section{Graft tension on second-look arthroscopy}

Twenty-two of the total studies evaluated the tension of the graft by probing during second-look arthroscopy. In addition, most studies objectively evaluated the graft tension using physical examination, Telos stress radiography, and KT-arthrometer. The most common classification of graft tension also involved three categories depending on how the graft moved during probing: taut, slightly (mildly) lax, and lax. Nine studies used these categories $[5,8,10,18,25,27-29,34]$. "Taut" is the same tension as normal ACL when moving within $3 \mathrm{~mm}$ of tension or probing. "Slightly lax" is laxer than normal ACL, moving $3 \mathrm{~mm}$ or more when probing but with a firm endpoint felt. Finally, "lax" is used in cases where 
Table 1 Selected study characteristics

\begin{tabular}{|c|c|c|c|c|c|c|c|}
\hline & $\begin{array}{l}\text { Year of publication } \\
\text { (journal) }\end{array}$ & $\begin{array}{l}\text { Level of } \\
\text { evidence }\end{array}$ & $\begin{array}{l}\text { Mean age of } \\
\text { patients (year) }\end{array}$ & $\begin{array}{l}\text { Grafts } \\
\text { (number of cases) }\end{array}$ & Technique & Follow-up (months) & mCMS \\
\hline Defrere et al. [19] & $1994($ CORR) & IV & $32(18.9-70.8)$ & FL: 47 & $\pi, S B$ & 1 year after surgery & 41 \\
\hline Jung et al. [20] & 1997 (Bull Hosp Jt Dis) & IV & $28(20-38)$ & BT-AT: 19 & Not described, SB & BT-AT: 15.8 (12-28) & 39 \\
\hline Toritsuka et al. [5] & 2004 (Arthroscopy) & IV & $24(15-51)$ & HA: 156 & Not described, SB/DB & $\begin{array}{l}<12,12-24 \\
>24(5-50)\end{array}$ & 49 \\
\hline Iwai et al. [21] & 2005 (AOTS) & III & $24.1(15-44)$ & $\begin{array}{l}\text { BT-AT + ITB: } 43 \\
\text { BT-AT alone: } 45\end{array}$ & Not described, SB & $>24$ & 46 \\
\hline Ahn et al. [6] & 2007 (Arthroscopy) & IV & $29.1(15-54)$ & $\mathrm{HA}: 74$ & $\pi, \mathrm{SB}$ & $20.1(9-32)$ & 43 \\
\hline Ahn et al. [22] & 2007 (KSSTA) & III & $31(16-60)$ & $\begin{array}{l}\text { BT-AT: } 80 \\
\text { HA: } 129\end{array}$ & $\Pi$ T, SB & $21.2(14-70)$ & 49 \\
\hline Kondo et al. [7] & 2007 (Arthroscopy) & $\|$ & $27(13-62)$ & HA: 136 & $\Pi$ T, DB & $14(11-24)$ & 59 \\
\hline Otsubo et al. [23] & 2007 (KSSTA) & IV & $24.1(15-44)$ & HA: 65 & $\mathrm{TP}, \mathrm{DB}$ & $16.5(5-29)$ & 43 \\
\hline \multirow[t]{3}{*}{ Lee et al. [24] } & \multirow[t]{3}{*}{2010 (Arthroscopy) } & \multirow[t]{3}{*}{ IV } & BT-AL: 27.9 (13-60) & BT-AL: 16 & \multirow[t]{3}{*}{$\pi, \mathrm{SB}$} & BTB: 38 (17-69) & \multirow[t]{3}{*}{46} \\
\hline & & & TA: 28.6 (15-60) & TA: 47 & & TA: 33 (12-66) & \\
\hline & & & HA: 29.3 (15-52) & $\mathrm{HA}: 43$ & & HA: 27 (15-50) & \\
\hline Mae et al. [25] & 2010 (Arthroscopy) & IV & $31.2(15-52)$ & $\mathrm{HA}: 25$ & $\mathrm{Ol}, \mathrm{DB}$ & $13.9(10-21)$ & 39 \\
\hline Ahn et al. [26] & 2011 (JBJS) & IV & $31(15-58)$ & $\mathrm{HA}: 37$ & $\pi, \mathrm{DB}$ & $>24$ & 39 \\
\hline Ahn et al. [27] & 2011 (Arthroscopy) & IV & $32.2(17-54)$ & $\mathrm{HA}: 33$ & $\Pi$ T, SB & $>12$ & 39 \\
\hline \multirow[t]{3}{*}{ Kinugasa et al. [8] } & \multirow[t]{3}{*}{2011 (Arthroscopy) } & \multirow[t]{3}{*}{$\|$} & \multirow[t]{3}{*}{$30.9(14-71)$} & HA (A): 55 & \multirow[t]{3}{*}{$\mathrm{Ol}, \mathrm{DB}$} & HA (A): $14.3(11-20)$ & \multirow[t]{3}{*}{56} \\
\hline & & & & $\mathrm{HA}(\mathrm{B}): 36$ & & HA (B): 13.1 (8-20) & \\
\hline & & & & $\mathrm{HA}(\mathrm{C}): 11$ & & HA (C): 15.5 (12-20) & \\
\hline \multirow[t]{2}{*}{ Ohsawa et al. [28] } & \multirow[t]{2}{*}{2012 (AJSM) } & \multirow[t]{2}{*}{ III } & AM: 24.9 (15-50) & HA: 99 & \multirow[t]{2}{*}{ TP/OI, DB } & \multirow[t]{2}{*}{1 year after surgery } & \multirow[t]{2}{*}{46} \\
\hline & & & PL: 24.1 (14-46) & & & & \\
\hline Ohsawa et al. [29] & 2012 (Arthroscopy) & IV & $36.8(15-57)$ & HA: 19 & TP, selective SB & 1 year after surgery & 39 \\
\hline Tanaka et al. [30] & 2012 (KSSTA) & IV & $25.5 \pm 8.5$ & $\mathrm{HA}: 41$ & $\mathrm{TP}, \mathrm{TB}$ & $6-22$ & 43 \\
\hline \multirow[t]{2}{*}{ Kim et al. [31] } & \multirow[t]{2}{*}{2014 (Knee) } & \multirow[t]{2}{*}{ III } & RP: $28.9 \pm 8.8$ & HA (RP): 36 & \multirow[t]{2}{*}{$\Pi \mathrm{T}, \mathrm{SB}$} & $\mathrm{HA}(\mathrm{RP}): 27.5 \pm 3.1$ & \multirow[t]{2}{*}{43} \\
\hline & & & RS: $32.0 \pm 9.4$ & HA (RS): 30 & & $\mathrm{HA}(\mathrm{RS}): 26.6 \pm 2.1$ & \\
\hline \multirow[t]{4}{*}{ Nakamae et al. [32] } & \multirow[t]{4}{*}{2014 (BJJ) } & \multirow[t]{4}{*}{ III } & SB: $24.6 \pm 11.9$ & $\mathrm{HA}(\mathrm{SB}): 61$ & Not described, & HA (SB): 25.6 (18-72) & 53 \\
\hline & & & DB: $24.8 \pm 11.0$ & $\mathrm{HA}(\mathrm{DB}): 82$ & & & \\
\hline & & & AG: $26.6 \pm 11.4$ & HA (AG): 73 & & HA (DB): $24.2(18-41)$ & \\
\hline & & & & & & HA (AG): 23.1 (18-72) & \\
\hline Ahn et al. [9] & 2015 (Arthroscopy) & III & PA: 37.2 (19-61) & HA (PA): 88 & $\mathrm{TP}, \mathrm{DB}$ & HA (PA): $45.0(25-77)$ & 52 \\
\hline & & & NA: 34.7 (15-57) & HA (NA): 66 & & HA (NA): 45.5 (25-87) & \\
\hline Kondo et al. [17] & 2015 (AJSM) & $\|$ & $29(13-58)$ & HA (RP): 62 & $\pi, \mathrm{DB}$ & $14(11-24)$ & 56 \\
\hline & & & & HA (RS): 46 & & & \\
\hline Lu et al. [18] & 2015 (AJSM) & $\|$ & $26.3(18-32)$ & $\mathrm{HA}(\mathrm{EF}): 28$ & $\mathrm{TP}, \mathrm{DB}$ & HA (EF): $80.6 \pm 16.3$ & 61 \\
\hline & & & & $\mathrm{HA}(\mathrm{BL}): 31$ & & $\mathrm{HA}(\mathrm{BL}): 65.2 \pm 12.8$ & \\
\hline Choi et al. [33] & 2017 (Knee) & III & $29.1(15-54)$ & $\mathrm{HA} / \mathrm{ACH}(\mathrm{RP}): 61$ & $\Pi$ T, SB & $\begin{array}{l}\text { HA/ACH (RP): } \\
14.8(10-23)\end{array}$ & 46 \\
\hline & & & & HA/ACH (RS): 30 & & $\begin{array}{l}\text { HA/ACH (RS): } \\
15.1(10-25)\end{array}$ & \\
\hline Kim et al. [10] & 2017 (KSSTA) & III & RP: $30.3 \pm 11.6$ & QA (RP): 42 & $\Pi$ T, SB & $\mathrm{QA}(\mathrm{RP}): 17.9 \pm 6.4$ & 43 \\
\hline & & & RS: $26.3 \pm 8.4$ & QA (RS): 33 & & QA (RS): $18.2 \pm 5.8$ & \\
\hline Kim et al. [11] & 2017 (KSSTA) & III & TA: $30.8 \pm 13.3$ & TA: 30 & TP/OI, SB & TA: 22.5 & 47 \\
\hline & & & $\mathrm{HA}: 28.9 \pm 10.1$ & HA: 26 & & HA: 22.5 & \\
\hline Matsushita et al. [12] & 2017 (KSSTA) & IV & PL: $35.1 \pm 16.4$ & HA (PL): 16 & $\mathrm{TP}, \mathrm{SB} / \mathrm{DB}$ & 1 year after surgery & 43 \\
\hline
\end{tabular}


Table 1 Selected study characteristics (Continued)

\begin{tabular}{|c|c|c|c|c|c|c|c|}
\hline & $\begin{array}{l}\text { Year of publication } \\
\text { (journal) }\end{array}$ & $\begin{array}{l}\text { Level of } \\
\text { evidence }\end{array}$ & $\begin{array}{l}\text { Mean age of } \\
\text { patients (year) }\end{array}$ & $\begin{array}{l}\text { Grafts } \\
\text { (number of cases) }\end{array}$ & Technique & Follow-up (months) & mCMS \\
\hline & & & SB: $30.1 \pm 10.5$ & HA (DB): 37 & & & \\
\hline \multirow[t]{2}{*}{ Nakayama et al. [13] } & 2017 (Knee) & III & PR: 26.6 (14-55) & HA (PR): 14 & Ol, DB & 1 year after surgery & 39 \\
\hline & & & RS: 26.4 (12-59) & HA (RS): 22 & & & \\
\hline \multirow[t]{2}{*}{ Xu et al. [34] } & 2017 (Med Sci Monit) & III & HA: $32.8 \pm 8.9$ & HA: 31 & $\mathrm{TP}, \mathrm{SB}$ & $\mathrm{HA}: 28.4 \pm 2.1$ & 43 \\
\hline & & & $H Y: 33.9 \pm 8.4$ & HY: 37 & & $H Y: 28.3 \pm 2.8$ & \\
\hline \multirow[t]{2}{*}{ Yoo et al. [14] } & 2017 (KSSTA) & I & TA: 24 (13-52) & TA: 25 & $\mathrm{TP}, \mathrm{SB}$ & TA: 34.5 (25.3-59.5) & 58 \\
\hline & & & HA: 30 (15-62) & HA: 26 & & HA: 32.8 (28.7-50.1) & \\
\hline
\end{tabular}

$m C M S$ modified Coleman Methodology Scores, FL fascia lata allograft, $B T$-AT bone patella bone tendon autograft, $B T-A L$ bone patella bone tendon allograft, $T A$ tibialis allograft, $H A$ hamstring autograft, $H Y$ hybrid graft, $Q A$ quadriceps autograft, $A C H$ Achilles allograft, $T$ transtibial technique, $T P$ transportal technique, $O$ I outside-in technique, $S B$ single bundle reconstruction, $A G$ single bundle augmentation, $D B$ double bundle reconstruction, $T B$ triple bundle reconstruction, $P L$ posterolateral bundle reconstruction, $R P$ remnant preservation, $R S$ remnant sacrifice, $P A$ provisional anatomic reconstruction, $N A$ non-anatomical reconstruction, $A M$ anteromedial, $P L$ posterolateral

CORR Clinical Orthopaedics and Related Research, Bull Hosp Jt Dis Bulletin of the Hospital for Joint Diseases, AOTS Archives of Orthopaedic and Trauma Surgery, KSSTA Knee Surgery, Sports Traumatology, Arthroscopy, JBJS Journal of Bone \& Joint Surgery, AJSM The American Journal of Sports Medicine, BJJ The Bone \& Joint Journal, Med Sci Monit Medical Science Monitor

the tension of the graft is not felt and on probing moves $10 \mathrm{~mm}$ or more. Five studies classified graft tension into two categories (normal and abnormal) [6, 21-23, 30], whereas the other two studies classified it into four categories according to their own criteria $(<2$ or $3 \mathrm{~mm}, 2$ or $3 \mathrm{~mm}$ to $5 \mathrm{~mm}, 5$ to $10 \mathrm{~mm},>10 \mathrm{~mm}$ ) [9, 24].

\section{Synovial coverage of graft on second-look arthroscopy}

Most studies (26/28) used synovial coverage to evaluate graft maturation in second-look arthroscopy. The most common classification of synovial coverage involved three categories. However, the criteria for assigning the categories vary by study. Kondo et al. [7] classified graft maturation as completely covered, partially covered, and almost not covered according to synovial coverage of the graft. Kinugasa et al. [8] classified it according to the synovial coverage percentage-more than $80 \%$ as good, less than $50 \%$ as poor, and intermediate as fair-while Kim et al. [11] used values of $75 \%, 25-75 \%$, and $25 \%$, respectively. Among the studies that classified synovial coverage into two categories, Otsubo et al. [23] referred to more than $50 \%$ of synovial coverage as normal, but Yoo et al. [14] considered more than $20 \%$ of synovial coverage as good. Some studies used two [14, 22, 24] or four categories [9, $33,34]$ to evaluate the synovial coverage of the graft.

\section{Neo-vascularization on second-look arthroscopy}

Only three of the included studies used neo-vascularization for evaluating graft maturation $[9,11,18]$. Ahn et al. [9] classified synovial coverage with re-vascularization and assigned scores as follows: synovial coverage $\geq 75 \%$ and abundant re-vascularization (4 points); synovial coverage $\geq 75 \%$ but lack of revascularization ( 3 points); synovial coverage of $50-75 \%$ ( 2 points); synovial coverage of $25-50 \%$ ( 1 point); and synovial coverage $<25 \%$ ( 0 points). $\mathrm{Lu}$ et al. also combined synovial coverage and vascularization classifying into 3 categories [18]. Kim et al. [11] evaluated the vascular coverage of grafts using values of $>75 \%, 25-75 \%$, and $<25 \%$.

\section{Total graft maturation score and grading system}

Seven studies tried to evaluate graft maturation comprehensively by combining various parameters. They calculated the total graft maturation score by summing up the scores assigned to each evaluation parameter. Details of each study are summarized in Table 3. The Kondo and Yasuda were the first to use the total graft maturation score and grading system [7], and several studies have used this method to evaluate the maturation of grafts [12, 17]. Ahn et al. [9] evaluated three parameters, graft integrity, tension, and synovial coverage with revascularization. They compared the graft maturation score and clinical outcomes between the anatomic reconstruction group and non-anatomic reconstruction group. Kim et al. [11] also used total graft maturation score to compare graft maturation between hamstring autografts and tibialis allografts. However, only one of the seven studies investigated the correlation between total maturation score and clinical outcome [7].

\section{Correlation between the second-look findings and clinical outcomes}

Nine of the included studies compared second-look findings with clinical outcomes (Table 4). Most studies (8/9) compared the results of the second-look findings and objective stability (KT-arthrometer, physical examination). With regard to stability, two studies reported that graft tension in the second-look arthroscopy was correlated with objective stability $[5,22]$, but this was not the case in other studies $[6,23,32]$. However, graft integrity $[5,22,30,32]$ and synovial coverage $[22,30,32]$ had no correlation with stability in included studies. 
Table 2 Summary of parameters and categories used for evaluating graft maturation on second-look arthroscopy

\begin{tabular}{|c|c|c|c|}
\hline & Integrity & Tension & Synovial coverage \\
\hline \multirow{4}{*}{$\begin{array}{l}\text { Defrere et al. [19] } \\
\text { (CORR, 1994) }\end{array}$} & \multicolumn{3}{|c|}{ 1. Invested either by a synovial layer or by neovascularization } \\
\hline & \multicolumn{3}{|l|}{ - Perfect stability; slight instability } \\
\hline & \multicolumn{3}{|c|}{ 2. Non-invested (without sign of above) } \\
\hline & \multicolumn{3}{|c|}{ - Perfect stability; slight instability; rupture in one half; graft failure } \\
\hline \multirow{3}{*}{$\begin{array}{l}\text { Jung YB et al. [20] } \\
\text { (Bull Hosp Jt Dis, 1997) }\end{array}$} & \multicolumn{3}{|c|}{ One or more of the following findings: } \\
\hline & \multirow{2}{*}{\multicolumn{3}{|c|}{$\begin{array}{l}\text { Nearly normal appearance; incomplete synovial coverage; partially torn fibers at the femoral tunnel site; } \\
\text { parallel fragmentation with cyclops lesion; impingement without the damage of the ACL graft }\end{array}$}} \\
\hline & & & \\
\hline \multirow{5}{*}{$\begin{array}{l}\text { Toritsuka et al. [5] } \\
\text { (AOTS, 2005) }\end{array}$} & No tear; & Arthroscopic probe test & NA \\
\hline & Partial tear & Taut (as normal ACL); & \\
\hline & - Superficial partial tear & Mildly lax (less tension, & \\
\hline & - Substantial partial tear & showing redundancy); & \\
\hline & - Partial tear, not classified & Lax (obvious loss of tension) & \\
\hline \multirow{3}{*}{$\begin{array}{l}\text { Iwai et al. [21] } \\
\text { (AOTS, 2005) }\end{array}$} & Fibrous split (+) & Arthroscopic probe test & NA \\
\hline & \multirow[t]{2}{*}{ Fibrous split (-) } & Normal tension; & \\
\hline & & Decreased tension & \\
\hline \multirow{3}{*}{$\begin{array}{l}\text { Ahn et al. [6] } \\
\text { (Arthroscopy, 2007) }\end{array}$} & Normal; & Arthroscopic probe test & Good (complete) \\
\hline & Partial tear & Taut; slightly lax & Half (insufficient) \\
\hline & \multicolumn{2}{|l|}{ Complete tear } & Pale (severely insufficient) \\
\hline \multirow{3}{*}{$\begin{array}{l}\text { Ahn et al. [22] } \\
\text { (KSSTA, 2007) }\end{array}$} & Normal; & Arthroscopic probe test & Fair (well covered) \\
\hline & Partial tear; & Taut; slightly lax & Poor (barely covered) \\
\hline & \multicolumn{3}{|l|}{ Complete tear } \\
\hline \multirow{3}{*}{$\begin{array}{l}\text { Kondo et al. [7] } \\
\text { (Arthroscopy, 2007) }\end{array}$} & \multicolumn{2}{|c|}{ A (no laceration or elongation of thick graft) } & A (completely covered) \\
\hline & \multicolumn{2}{|c|}{$\begin{array}{l}\text { B (partial laceration of thick graft or no laceration or elongation } \\
\text { of thin graft) }\end{array}$} & B (partial) \\
\hline & \multicolumn{2}{|c|}{ C (complete tear or obvious elongation) } & C (almost not) \\
\hline \multirow{3}{*}{$\begin{array}{l}\text { Otsubo et al. [23] } \\
\text { (KSSTA, 2007) }\end{array}$} & No rupture & Arthroscopic probe test & Good (whole length) \\
\hline & Partial rupture & Taut (normal ACL) & Fair $(>50 \%)$ \\
\hline & Total rupture & Lax (loss of tension) & Poor $(<50 \%)$ \\
\hline \multirow{5}{*}{$\begin{array}{l}\text { Lee et al. [24] } \\
\text { (Arthroscopy, 2010) }\end{array}$} & Normal & Arthroscopic probe test & Normal (> 50\%) \\
\hline & \multirow[t]{4}{*}{ Abnormal (tear or cyclops lesion) } & Normal (<3 mm) & \multirow[t]{4}{*}{ Abnormal (<50\%) } \\
\hline & & Nearly normal (3-5 mm) & \\
\hline & & Abnormal (5-10 mm) & \\
\hline & & Severely abnormal (> 10 mm) & \\
\hline \multirow{3}{*}{$\begin{array}{l}\text { Mae et al. [25] } \\
\text { (Arthroscopy, 2010) }\end{array}$} & No tear; & Arthroscopic probe test & Good (whole length) \\
\hline & Superficial tear & Taut; mildly lax; lax & Fair $(>50 \%)$ \\
\hline & Substantial tear & & Poor $(<50 \%)$ \\
\hline \multirow{3}{*}{$\begin{array}{l}\text { Ahn et al. [26] } \\
(J B J S, 2011)\end{array}$} & A (no laceration or elongation of $t$ & & A (complete) \\
\hline & B (partial laceration of thick graft $\mathrm{C}$ & ration/elongation of thin graft) & B (partial) \\
\hline & C (complete tear or obvious elong & & C (almost not) \\
\hline Ahn et al. [27] & No tear & Arthroscopic probe test & Good \\
\hline (Antriroscopy, zUII) & Partial tear & Taut; mildly lax; lax & Fair \\
\hline & Complete tear & & Poor \\
\hline Kinugasa et al. [8] (Arthroscopy, 2011) & No tear & Arthroscopic probe test & Good (> 80\%) \\
\hline & Superficial tear & Taut; mildly lax; lax & Fair (50-80\%) \\
\hline
\end{tabular}


Table 2 Summary of parameters and categories used for evaluating graft maturation on second-look arthroscopy (Continued)

\begin{tabular}{|c|c|c|c|}
\hline & Integrity & Tension & Synovial coverage \\
\hline & Substantial tear & & Poor $(<50 \%)$ \\
\hline \multirow{4}{*}{$\begin{array}{l}\text { Ohsawa et al. [28] } \\
\text { (AJSM, 2012) }\end{array}$} & No tear (> 80\%) & Arthroscopic probe test & Excellent (entire coverage) \\
\hline & Partial tear (30-80\%) & $\begin{array}{l}\text { Taut }(<3 \mathrm{~mm} \text {, firm end } \\
\text { point })\end{array}$ & Fair (partial defect) \\
\hline & Complete tear $(<30 \%)$ & $\begin{array}{l}\text { Slightly lax (> } 3 \mathrm{~mm} \text {, firm } \\
\text { end point) }\end{array}$ & Poor (barely covered) \\
\hline & & Lax (no firm end point) & \\
\hline \multirow{4}{*}{$\begin{array}{l}\text { Ohsawa et al. [29] } \\
\text { (Arthroscopy, 2012) }\end{array}$} & No tear (> 80\%) & Arthroscopic probe test & Excellent (entire coverage) \\
\hline & Partial tear (30-80\%) & $\begin{array}{l}\text { Taut }(<3 \mathrm{~mm} \text {, firm } \\
\text { end point) }\end{array}$ & Fair (partial defect) \\
\hline & Complete tear $(<30 \%)$ & $\begin{array}{l}\text { Slightly lax (> } 3 \mathrm{~mm} \text {, firm } \\
\text { end point) }\end{array}$ & Poor (barely covered) \\
\hline & & Lax (no firm end point) & \\
\hline \multirow{3}{*}{$\begin{array}{l}\text { Tanaka et al. [30] } \\
\text { (KSSTA, 2012) }\end{array}$} & No tear & Arthroscopic probe test & Good (whole length covered) \\
\hline & Substantial tear & Taut (as normal ACL) & Fair (> 50\%) \\
\hline & & Lax (looser than normal) & Poor $(<50 \%)$ \\
\hline \multirow{3}{*}{$\begin{array}{l}\text { Kim et al. [31] } \\
\text { (Knee, 2014) }\end{array}$} & No tear & No arthroscopic test & Good \\
\hline & Partial tear & (tested by KT-2000 & Partial \\
\hline & Complete tear & & Poor \\
\hline \multirow{4}{*}{$\begin{array}{l}\text { Nakamae et al. [32] } \\
\text { (BJJ, 2014) }\end{array}$} & Normal & & 1. Synovial coverage of graft: \\
\hline & Damaged (obvious lack of tension or a su & tantial tear) & $\begin{array}{l}\text { good (>80\%); fair (50-80\%); } \\
\text { poor (<50\%) }\end{array}$ \\
\hline & & & $\begin{array}{l}\text { 2. Synovial coverage between } \\
\text { the graft and the femoral } \\
\text { bone tunnel: }\end{array}$ \\
\hline & & & good $(>80 \%)$; poor $(<80 \%)$ \\
\hline \multirow{2}{*}{$\begin{array}{l}\text { Ahn et al. [9] } \\
\text { (Arthroscopy, 2015) }\end{array}$} & $\geq 90 \% ; 75-90 \% ; 50-75 \% ;<50 \%$ & Arthroscopic probe test & $\geq 75 \% ;$ 50-75\%; 25-50\%; <25\% \\
\hline & & $\begin{array}{l}<2 \mathrm{~mm} ; 2-5 \mathrm{~mm} ; 5-10 \mathrm{~mm} ; \\
>10 \mathrm{~mm}\end{array}$ & \\
\hline \multirow{3}{*}{$\begin{array}{l}\text { Kondo et al. [17] } \\
\text { (AJSM, 2015) }\end{array}$} & I (no laceration or tear) & No arthroscopic test & I (completely with thick tissue) \\
\hline & II (partial laceration) & (tested by KT-2000 & II (completely with thin tissue) \\
\hline & III (complete tear or obvious elongation) & and PST) & $\begin{array}{l}\text { III (partly with thin tissue or almost } \\
\text { not covered) }\end{array}$ \\
\hline \multirow[t]{4}{*}{$\begin{array}{l}\text { Lu et al. [18] } \\
(\text { AJSM, 2015) }\end{array}$} & No tear (> 80\%) & Arthroscopic probe test & $\begin{array}{l}\text { Synovial coverage and } \\
\text { vascularization }\end{array}$ \\
\hline & Partial tear (30-80\%) & Taut (tense as normal ACL) & Excellent (entirely covered) \\
\hline & Complete tear $(<30 \%)$ & $\begin{array}{l}\text { Mildly lax (less tension, } \\
\text { redundant) }\end{array}$ & Fair (partial defect) \\
\hline & & $\begin{array}{l}\text { Lax (obvious loss } \\
\text { of tension) }\end{array}$ & Poor (barely covered) \\
\hline \multirow[t]{4}{*}{$\begin{array}{l}\text { Choi et al. [33] } \\
\text { (Knee, 2017) }\end{array}$} & N/A & $\begin{array}{l}\text { Arthroscopic probe } \\
\text { test and }\end{array}$ & Excellent (> 80\%) \\
\hline & & $\begin{array}{l}\text { intra-operative } \\
\text { anterior drawer test }\end{array}$ & Good (50-80\%) \\
\hline & & (translation was & Fair (20-50\%) \\
\hline & & measured by ruler) & Poor $(<20 \%)$ \\
\hline \multirow{3}{*}{$\begin{array}{l}\text { Kim et al. [10] } \\
\text { (KSSTA, 2017) }\end{array}$} & I (> 80\% intact) & Arthroscopic probe test & I (> 80\%) \\
\hline & $\|(<80 \%$ intact $)$ & I (<3 mm, firm end $)$ & || (30-80\%) \\
\hline & III (<30\% intact) & II (> 3 mm, firm end); & III (<30\%) \\
\hline
\end{tabular}


Table 2 Summary of parameters and categories used for evaluating graft maturation on second-look arthroscopy (Continued)

\begin{tabular}{|c|c|c|c|}
\hline & Integrity & Tension & Synovial coverage \\
\hline & & III (no firm end) & \\
\hline \multirow{3}{*}{$\begin{array}{l}\text { Kim et al. }[11]^{\dagger} \\
(\text { KSSTA, 2017) }\end{array}$} & Complete tear & No arthroscopic test & $>75 \%$ \\
\hline & Partial tear & (tested by Lachman test and & $25-75 \%$ \\
\hline & No tear & stress radiograph) & $<25 \%$ \\
\hline \multirow{3}{*}{$\begin{array}{l}\text { Matsushita et al. } \\
\text { [12] (KSSTA, 2017) }\end{array}$} & No laceration & No elongation & Completely covered \\
\hline & Partial tear & Elongation of thin graft & Partially covered \\
\hline & Complete tear & Obvious elongation & Almost not covered \\
\hline $\begin{array}{l}\text { Nakayama et al. } \\
\text { [13] (Knee, 2017) }\end{array}$ & $\begin{array}{l}\text { Good (no partial tear); } \\
\text { fair (superficial tear); poor (substantial tear) }\end{array}$ & $\begin{array}{l}\text { No arthroscopic test } \\
\text { (tested by KT-1000) }\end{array}$ & $\begin{array}{l}\text { Good (complete); } \\
\text { fair (> 50\%); poor }(<50 \%)\end{array}$ \\
\hline \multirow{3}{*}{$\begin{array}{l}\text { Xu et al. [34] } \\
\text { (Med Sci Monit, 2017) }\end{array}$} & Good & Arthroscopic probe test & $>75 \% ;$ 50-75\%; 25-50\%; <25\% \\
\hline & Fair & Taut; mildly lax; lax & \\
\hline & Damaged (re-tear) & & \\
\hline \multirow{2}{*}{$\begin{array}{l}\text { Yoo et al. [14] ( } \\
\text { KSSTA, 2017) }\end{array}$} & Intact & No arthroscopic test & Good (> 20\%) \\
\hline & Partial tear; total tear & $\begin{array}{l}\text { (tested by Lachman } \\
\text { test and PST) }\end{array}$ & Poor $(<20 \%)$ \\
\hline
\end{tabular}

†Three studies also evaluated neo-vascularization of grafts. PST pivot-shift test

CORR Clinical Orthopaedics and Related Research, Bull Hosp Jt Dis Bulletin of the Hospital for Joint Diseases, AOTS Archives of Orthopaedic and Trauma Surgery, KSSTA Knee Surgery, Sports Traumatology, Arthroscopy, JBJS Journal of Bone \& Joint Surgery, AJSM The American Journal of Sports Medicine, BJJ The Bone \& Joint Journal, Med Sci Monit Medical Science Monitor

Table 3 Summary of the Studies that Used Total Maturation Score or Grading System

\begin{tabular}{lll}
\hline & Method & Correlation with clinical outcomes \\
\hline $\begin{array}{l}\text { Kondo et al. [7] (Arthroscopy, } \\
\text { 2007) }\end{array}$ & Graft maturation score for each bundle based on & Category I showed significantly better \\
& graft tension $(0-2)^{*}$ and integrity $(0-2)$. Each bundle & results than categories II or III in KT-2000 \\
& and PST \\
& excellent, 2 or 3 as fair, and 0 or 1 as poor. DB & No significant differences between the \\
& grafts were classified into categories I & three categories in all clinical outcomes. \\
& (two excellent bundles), II (only one excellent & \\
& bundle), or III (no excellent bundle)
\end{tabular}

Ahn et al. [9] (Arthroscopy, Graft maturation score based on integrity (0-3), 2015) tension (0-3), and synovial coverage with neo-vascularization (0-4). Total graft maturation scores ranged from 0 to 10 points

Kondo et al. [17] (AJSM, 2015) Graft maturation score and grade system by Kondo and Yasuda were used

$\begin{array}{ll}\text { Lu et al. [18] (AJSM, 2015) } & \text { Graft maturation score based on synovial and } \\ & \text { vascular coverage, tension, integrity. A maximum } \\ & \text { of } 2 \text { points was assigned for each parameter. } \\ & \text { A graft with a total score of } 5 \text { or } 6 \text { was evaluated } \\ & \text { as excellent, } 3 \text { or } 4 \text { as fair, and } \leq 2 \text { as poor }\end{array}$

Kim et al. [10] (KSSTA, 2017) Total maturation score based on graft tension, integrity, and synovial coverage (modified from method by Ohsawa et al. [28]). According to the second-look finding, a maximum of 3 points was assigned for each parameter. Total scores ranged from 3 to 9 points

Kim et al. [11] (KSSTA, 2017) Total maturation score based on graft tension, integrity, synovial coverage, and revascularization. According to the second-look finding, a maximum of 2 points was assigned for each parameter. Total scores ranged from 0 to 8 points

Matsushita et al. [12] (KSSTA, Graft maturation grade and category system by Kondo 2017)

\begin{abstract}
No comparison between second-look findings and objective outcomes, but remnant preserved group showed higher graft maturation score and better
\end{abstract} clinical outcomes

No comparison between second-look findings and objective outcomes, but remnant preserved group showed better maturation grade and stability (KT-2000, PST)

No comparison between second-look findings and objective clinical outcomes. But ACLR using the existing footprint remnant for tunnel placement showed higher graft maturation score and better functional results (ROM recovery, subjective outcome scores)

No comparison between total maturation score and clinical outcomes

No comparison between total maturation score and clinical outcomes

No comparison between total maturation grade/category and clinical outcomes

*Assigned score for each parameter in blank. $A M$ anteromedial bindle, $P L$ posterolateral bundle, $D B$ double bundle, $A C L R$ anterior cruciate ligament reconstruction AJSM The American Journal of Sports Medicine, KSSTA Knee Surgery, Sports Traumatology, Arthroscopy 
Table 4 Summary of the studies that compare second-look findings with clinical outcomes

\begin{tabular}{|c|c|c|c|}
\hline & Second-look findings & $\begin{array}{l}\text { Clinical outcomes compared } \\
\text { with second-look findings }\end{array}$ & Comments \\
\hline \multirow[t]{2}{*}{$\begin{array}{l}\text { Defrere et al. [19] (CORR, } \\
\text { 1994) }\end{array}$} & \multirow{2}{*}{$\begin{array}{l}\text { Invested or non-invested } \\
\text { (based on synovial coverage } \\
\text { and neovascularization) }\end{array}$} & \multirow[t]{2}{*}{ Stability (Lachman test, PST), failure } & $\begin{array}{l}\text { Invested (25): perfect stability (21), slight } \\
\text { instability (4) }\end{array}$ \\
\hline & & & $\begin{array}{l}\text { Non-invested (22): perfect stability (13), slight } \\
\text { instability (4), graft rupture in one half (1), Failure (4) }\end{array}$ \\
\hline \multirow{2}{*}{$\begin{array}{l}\text { Toritsuka et al. [5] } \\
\text { (AOTS, 2005) }\end{array}$} & \multirow{2}{*}{$\begin{array}{l}\text { Tension (probe test), } \\
\text { integrity (tear) }\end{array}$} & \multirow[t]{2}{*}{ KT-1000 (STSD) } & $1.1 \mathrm{~mm}$ (taut) $\vee 2.3 \mathrm{~mm}$ (mildly lax/lax), $p=0.003$ \\
\hline & & & $\begin{array}{l}1.2 \mathrm{~mm} \text { (minimal tear) } \vee 1.2 \mathrm{~mm} \\
\text { (substantial tear), } p=0.670\end{array}$ \\
\hline $\begin{array}{l}\text { Ahn et al. [6] } \\
\text { (Arthroscopy, 2007) }\end{array}$ & Tension (probe test) & KT-2000 & $\begin{array}{l}\text { No statistical difference in KT-2000 measurements } \\
\text { between "good tension" and "some laxity" group }\end{array}$ \\
\hline \multirow[t]{2}{*}{$\begin{array}{l}\text { Ahn et al. [22] } \\
\text { (KSSTA, 2007) }\end{array}$} & \multirow[t]{2}{*}{$\begin{array}{l}\text { Tension (probe test), tear, } \\
\text { synovial coverage, notch } \\
\text { reformation, cyclops lesion }\end{array}$} & \multirow[t]{2}{*}{$\begin{array}{l}\text { KT-2000 } \\
\text { Lysholm score }\end{array}$} & $\begin{array}{l}\text { "Normal tension" group was significantly better than } \\
\text { "slightly lax" group in KT-2000, but there was no } \\
\text { significant difference in Lysholm score between groups }\end{array}$ \\
\hline & & & $\begin{array}{l}\text { Graft tear, synovial coverage, notch reformation, and } \\
\text { cyclops-like lesion showed no clinical correlation with } \\
\text { KT-2000 and Lysholm score. }\end{array}$ \\
\hline \multirow[t]{2}{*}{$\begin{array}{l}\text { Kondo et al. [7] } \\
\text { (Arthroscopy, 2007) }\end{array}$} & \multirow[t]{2}{*}{$\begin{array}{l}\text { Three categories based } \\
\text { on maturation score }\end{array}$} & \multirow{2}{*}{$\begin{array}{l}\text { Stability (KT-2000, PST) Clinical } \\
\text { outcomes (ROM, Lysholm and IKDC } \\
\text { score, mean isokinetic peak torque) }\end{array}$} & $\begin{array}{l}\text { Category I showed significantly better results than } \\
\text { categories II or III in KT-2000 and PST }\end{array}$ \\
\hline & & & $\begin{array}{l}\text { No significant differences between the three } \\
\text { categories in all clinical outcomes }\end{array}$ \\
\hline $\begin{array}{l}\text { Otsubo et al. [23] } \\
\text { (KSSTA, 2007) }\end{array}$ & $\begin{array}{l}\text { Three groups based on } \\
\text { tension (probe test) and } \\
\text { integrity }\end{array}$ & KT-1000, PST & $\begin{array}{l}\text { No significant differences between the three groups } \\
\text { in KT-1000 and PST }\end{array}$ \\
\hline \multirow[t]{2}{*}{$\begin{array}{l}\text { Lee et al. [24] } \\
\text { (Arthroscopy, 2010) }\end{array}$} & \multirow[t]{2}{*}{$\begin{array}{l}\text { Synovial coverage, integrity, } \\
\text { tension (probe test), cyclops } \\
\text { or impingement }\end{array}$} & \multirow[t]{2}{*}{ IKDC grade } & $\begin{array}{l}\text { Normal synovial coverage group and normal tension } \\
\text { group had significantly more cases with IKDC } \\
\text { grade A/B than the abnormal groups }\end{array}$ \\
\hline & & & $\begin{array}{l}\text { Integrity and impingement/cyclops made no } \\
\text { difference in IKDC grade }\end{array}$ \\
\hline $\begin{array}{l}\text { Tanaka et al. [30] } \\
\text { (KSSTA, 2012) }\end{array}$ & $\begin{array}{l}\text { Graft damage and synovial } \\
\text { coverage of PL }\end{array}$ & $\begin{array}{l}\text { IKDC grade, Lachman test, PST, } \\
\text { KT arthrometer }\end{array}$ & $\begin{array}{l}\text { PL graft damage or synovial coverage showed no } \\
\text { significant correlation with objective outcomes }\end{array}$ \\
\hline $\begin{array}{l}\text { Nakamae et al. [32] } \\
\text { (BJJ, 2014) }\end{array}$ & $\begin{array}{l}\text { Graft condition (integrity and } \\
\text { tension), synovial coverage }\end{array}$ & KT-2000 and Lysholm score & $\begin{array}{l}\text { Graft condition and extent of synovial coverage did } \\
\text { not significantly affect the results of KT-2000 and } \\
\text { Lysholm score }\end{array}$ \\
\hline
\end{tabular}

PST pivot shift test, STSD side to side difference, ROM range of motion CORR Clinical Orthopaedics and Related Research, AOTS Archives of Orthopaedic and Trauma Surgery, KSSTA Knee Surgery, Sports Traumatology, Arthroscopy, BJJ The Bone \& Joint Journal

On the other hand, only three studies investigated the correlation between second-look findings and patientreported outcomes (IKDC subjective score, Lysholm score) $[7,22,32]$. All three of these studies reported that there was no correlation between second-look findings and patient-reported outcomes.

\section{Discussion}

This systematic review summarizes how graft maturation was evaluated on second-look arthroscopy following ACL reconstruction. All included studies evaluated graft maturation with two or more of the following three gross findings: graft integrity, tension, and synovial coverage. Two to four categories were used for evaluating each parameter, but the criteria for classification were slightly different for each study. Several studies also used the parameter of neo-vascularization to evaluate the viability of grafts.
Follow-up period from ACL reconstruction to secondlook arthroscopy varied according to the study, but the average length was about 1-2 years. Second-look arthroscopy to evaluate a graft should be performed only after the maturation of the graft ends. However, previous studies have reported different results on the timing of graft remodeling. Falconiero et al. [35] reported no significant differences in the histologic aspect of 12-month grafts evaluated by them. According to Sanchez et al. [36], the grafts reached maturity at around 2 years after surgery. Rougraff et al. [37] observed areas of degeneration, neo-vascularity, and hypercellularity until 3 years after reconstruction.

Graft integrity is the most basic evaluation method and almost all studies used integrity for evaluating graft maturation after ACL reconstruction. This was expressed as the presence and extent of the graft tear. In the majority of studies, graft integrity was classified into three categories: intact, partial (or superficial) tear, and complete (or 
substantial) tear. However, intact graft with no graft tears were mostly categorized as normal, whereas Ohsawa et al. classified normal integrity as over $80 \%[28,29]$ and several studies followed this classification $[10,18]$. The criteria for dividing the superficial tear and the substantial tear were also ambiguous, and some subjective judgment could be involved.

Tension of the graft is one of the most important evaluation parameters because it assesses the stability of the knee joint, which is the main purpose of ACL reconstruction. The tension of grafts was evaluated as taut, slightly lax, and lax by probing during second-look arthroscopy. This classification is problematic in that it depends on the subjective judgment of the examiner. Some studies described the use of rulers at the time of probing or the use of anatomical indicators such as femoral condyle to measure the tension of grafts, but this may also not be objective. In addition, the measurement of stability by arthroscopic probing has the disadvantage that it is difficult to evaluate the stability of rotation. Therefore, a method is needed that can be used to evaluate stability of rotation more objectively.

While the integrity and tension of grafts represent mechanical properties, synovial coverage has been used as a measure of biologic maturation. Synovial coverage of the graft was also mostly categorized into three categories, while the criteria for classification varied by study. Some studies regarded the best category as complete coverage $[6,7,18,28,30]$, but a few other studies classified it as more than $80 \%[8,10,32,33]$ or $50 \%$ [24] and some other studies as more than 20\% [14]. This seems to be influenced by the subjective philosophy of each surgeon as to how much synovial coverage is considered good. In addition, the method used for measuring the amount of synovial coverage has not always been clearly described. Therefore, consensus and unification of evaluation criteria for synovial coverage are required.

Recently, long term follow-up studies of ACL reconstruction have been published, and long-term survival of grafts is recognized as important. Bottoni et al. [2] reported higher long-term failure rates for allografts compared to autografts. The high long-term survival rate of autografts is possibly because only well-matured grafts can survive the stresses incurred with a high level of activity in the long-term. With regard to this, it seems to be important to evaluate graft viability, such as the neovascularization of grafts, although only a few studies did this on second-look arthroscopy $[9,11,18]$.

Several studies calculated the total maturation score by summing up the scores assigned to each evaluation parameter [7, 9-12, 17, 18]. Although these efforts have limitations that are not validated by the scoring system, it has the advantages of comparing graft maturation status between different graft types or surgical techniques or correlating graft maturation status with clinical outcomes. Kondo and Yasuda [7] reported that patients in the "excellent" group with higher total maturation score had better anteroposterior and rotational stability than those in the other groups. Ahn et al. [9] compared the anatomic reconstruction group with the non-anatomic reconstruction group and found that the former had better total graft maturation score and clinical outcomes. Kim et al. [11] demonstrated that total maturation scores of hamstring autografts were higher than those of tibialis allografts. In order to further develop such a total scoring system, efforts must be made to validate the results in comparison with clinical outcomes.

Although a few studies reported that graft tension in second-look arthroscopy was significantly correlated with objective stability test results $[5,22]$, second-look findings seem to be less correlated with clinical outcomes. Graft integrity $[5,22,30,32]$ and synovial coverage $[22,30,32]$ had no correlation with stability in the included studies. Also, there was no correlation between second-look findings and patient-reported outcomes [7, 22, 32]. These results may be due to the subjective evaluation of secondlook arthroscopy and the use of evaluation methods that have not yet been validated.

This study has several limitations. First, only five randomized trials, among 28 studies, were included for this systematic review. The quality of included studies was assessed to be relatively low (mean mCMS of $46.4 \pm 6.7$ ). Second, this study summarizes previous methods for evaluating graft maturation on second-look arthroscopy following ACL reconstruction, but it does not answer which evaluation method is appropriate. Consensus and validation are needed to determine which method is the most appropriate evaluation method. Third, the follow-up periods of the studies were relatively short and no longterm follow-up studies were included, and most studies had difficulty identifying the relationship between clinical outcomes and the evaluation of graft maturation. Finally, almost all the studies were from northeast Asia, including South Korea, Japan, and China; therefore, most studies are limited to specific races.

\section{Conclusions}

Graft integrity, tension, and synovial coverage were the most frequently evaluated criteria for graft maturation on second-look arthroscopy following ACL reconstruction. However, there is no uniform criterion for evaluation of each parameter. Therefore, development of a validated evaluation method for second-look arthroscopy is required.

\section{Abbreviations}

ACL: Anterior cruciate ligament; mCMS: Modified Coleman Methodology Scores; PRISMA: Preferred Reporting Items for Systematic Reviews and MetaAnalyses 


\section{Acknowledgements}

Not applicable.

\section{Funding}

This study received no funding to complete.

\section{Availability of data and materials}

This study has no associated data because it is a systematic review of previous studies.

\section{Authors' contributions}

SGK: manuscript writing, collection of data, data analysis and interpretation. $\mathrm{JHJ}$ : collection of data, data analysis and interpretation. JHS: collection of data. JHB: conception and design, final approval of the manuscript. This article was accepted in an old system (KSRR 19-007), not a new one. This article was guaranteed to be published by the new publisher without additional reviewing. The manuscript has never been published before nor is it under consideration for publication elsewhere. We are pleased to publish our study in Knee Surgery \& Related Research. Thank you. All authors read and approved the final manuscript.

\section{Competing interests}

The authors declare that they have no competing interests.

\section{Publisher's Note}

Springer Nature remains neutral with regard to jurisdictional claims in published maps and institutional affiliations.

\section{Author details}

${ }^{1}$ Department of Orthopaedic Surgery, Korea University Guro Hospital, Korea University College of Medicine, 148 Gurodong-ro, Guro-gu, Seoul 08308, Republic of Korea. ${ }^{2}$ Department of Rheumatology, Korea University Guro Hospital, Korea University College of Medicine, Seoul, Republic of Korea.

Received: 3 May 2019 Accepted: 10 May 2019

Published online: 28 June 2019

\section{References}

1. Amiel D, Kleiner JB, Roux RD et al (1986) The phenomenon of "ligamentization": anterior cruciate ligament reconstruction with autogenous patellar tendon. J Orthop Res 4:162-172

2. Bottoni CR, Smith EL, Shaha J et al (2015) Autograft versus allograft anterior cruciate ligament reconstruction: A prospective, randomized clinical study with a minimum 10-year follow-up. Am J Sports Med 43:2501-2509

3. Cvetanovich GL, Mascarenhas R, Saccomanno MF et al (2014) Hamstring autograft versus soft-tissue allograft in anterior cruciate ligament reconstruction: a systematic review and meta-analysis of randomized controlled trials. Arthroscopy 30:1616-1624

4. Lynch TS, Parker RD, Patel RM et al (2015) The Impact of the Multicenter Orthopaedic Outcomes Network (MOON) research on anterior cruciate ligament reconstruction and orthopaedic practice. Am Acad Orthop Surg 23:154-163

5. Toritsuka Y, Shino K, Horibe S et al (2004) Second-look arthroscopy of anterior cruciate ligament grafts with multistranded hamstring tendons. Arthroscopy 20:287-293

6. Ahn JH, Park JS, Lee YS, Cho YJ (2007) Femoral bioabsorbable crosspin fixation in anterior cruciate ligament reconstruction. Arthroscopy 23:1093-1099

7. Kondo E, Yasuda K (2007) Second-look arthroscopic evaluations of anatomic double-bundle anterior cruciate ligament reconstruction: relation with postoperative knee stability. Arthroscopy 23:1198-1209

8. Kinugasa K, Mae T, Matsumoto $\mathrm{N}$ et al (2011) Effect of patient age on morphology of anterior cruciate ligament grafts at second-look arthroscopy. Arthroscopy 27:38-45

9. Ahn JH, Kim JD, Kang HW (2015) Anatomic placement of the femoral tunnels in double-bundle anterior cruciate ligament reconstruction correlates with improved graft maturation and clinical outcomes. Arthroscopy 31:2152-2161

10. Kim BH, Kim Jl, Lee $\mathrm{O}$ et al (2017) Preservation of remnant with poor synovial coverage has no beneficial effect over remnant sacrifice in anterior cruciate ligament reconstruction. Knee Surg Sports Traumatol Arthrosc. https://doi.org/10.1007/s00167-017-4683-7

11. Kim SG, Kim SH, Kim JG et al (2017) Hamstring autograft maturation is superior to tibialis allograft following anatomic single-bundle anterior cruciate ligament reconstruction. Knee Surg Sports Traumatol Arthrosc. https://doi.org/10.1007/s00167-017-4649-9

12. Matsushita T, Kuroda R, Nishizawa $Y$ et al (2017) Clinical outcomes and biomechanical analysis of posterolateral bundle augmentation in patients with partial anterior cruciate ligament tears. Knee Surg Sports Traumatol Arthrosc 25:1279-1289

13. Nakayama H, Kambara S, Iseki T et al (2017) Double-bundle anterior cruciate ligament reconstruction with and without remnant preservation -

Comparison of early postoperative outcomes and complications. Knee 24: 1039-1046

14. Yoo SH, Song EK, Shin YR et al (2017) Comparison of clinical outcomes and second-look arthroscopic findings after $\mathrm{ACL}$ reconstruction using a hamstring autograft or a tibialis allograft. Knee Surg Sports Traumatol Arthrosc 25:1290-1297

15. Coleman BD, Khan KM, Maffulli N et al (2000) Studies of surgical outcome after patellar tendinopathy: clinical significance of methodological deficiencies and guidelines for future studies. Victorian Institute of Sport Tendon Study Group. Scand J Med Sci Sports 10:2-11

16. Wright JG, Swiontkowski MF, Heckman JD (2003) Introducing levels of evidence to the journal. J Bone Joint Surg Am 85-A:1-3

17. Kondo E, Yasuda K, Onodera J et al (2015) Effects of remnant tissue preservation on clinical and arthroscopic results after anatomic double-bundle anterior cruciate ligament reconstruction. Am J Sports Med 43:1882-1892

18. Lu W, Wang D, Zhu W et al (2015) Placement of double tunnels in ACL reconstruction using bony landmarks versus existing footprint remnant: a prospective clinical study with 2-year follow-up. Am J Sports Med 43:1206-1214

19. Defrere J, Franckart A (1994) Freeze-dried fascia lata allografts in the reconstruction of anterior cruciate ligament defects. A two- to seven-year follow-up study. Clin Orthop Relat Res 303:56-66

20. Jung YB, Yum JK (1997) Arthroscopic second look findings of an anterior cruciate ligament bone-patellar tendon-bone autograft. Bull Hosp Jt Dis 56: 154-160

21. Iwai M, Sugimoto K, Okahashi $K$ et al (2005) Effects of reinforcement with the iliotibial tract flap in $\mathrm{ACL}$ reconstruction using bone-patellar tendonbone. Arch Orthop Trauma Surg 125:228-237

22. Ahn JH, Yoo JC, Yang HS et al (2007) Second-look arthroscopic findings of 208 patients after ACL reconstruction. Knee Surg Sports Traumatol Arthrosc 15:242-248

23. Otsubo H, Shino K, Nakamura N et al (2007) Arthroscopic evaluation of $\mathrm{ACL}$ grafts reconstructed with the anatomical two-bundle technique using hamstring tendon autograft. Knee Surg Sports Traumatol Arthrosc 15:720-728

24. Lee JH, Bae DK, Song SJ et al (2010) Comparison of clinical results and secondlook arthroscopy findings after arthroscopic anterior cruciate ligament reconstruction using 3 different types of grafts. Arthroscopy 26:41-49

25. Mae T, Shino K, Matsumoto N et al (2010) Anatomic double-bundle anterior cruciate ligament reconstruction using hamstring tendons with minimally required initial tension. Arthroscopy 26:1289-1295

26. Ahn JH, Choi SH, Wang JH et al (2011) Outcomes and second-look arthroscopic evaluation after double-bundle anterior cruciate ligament reconstruction with use of a single tibial tunnel. J Bone Joint Surg Am 93:1865-1872

27. Ahn JH, Wang JH, Lee YS et al (2011) Anterior cruciate ligament reconstruction using remnant preservation and a femoral tensioning technique: Clinical and magnetic resonance imaging results. Arthroscopy 27:1079-1089+e1137

28. Ohsawa T, Kimura M, Hagiwara K et al (2012) Clinical and second-look arthroscopic study comparing 2 tibial landmarks for tunnel insertions during double-bundle $A C L$ reconstruction with a minimum 2-year follow-up. Am J Sports Med 40:2479-2486

29. Ohsawa T, Kimura M, Kobayashi Y et al (2012) Arthroscopic evaluation of preserved ligament remnant after selective anteromedial or posterolateral bundle anterior cruciate ligament reconstruction. Arthroscopy 28:807-817

30. Tanaka Y, Shino K, Horibe $S$ et al (2012) Triple-bundle ACL grafts evaluated by second-look arthroscopy. Knee Surg Sports Traumatol Arthrosc 20:95-101 
31. Kim MK, Lee SR, Ha JK et al (2014) Comparison of second-look arthroscopic findings and clinical results according to the amount of preserved remnant in anterior cruciate ligament reconstruction. Knee 21:774-778

32. Nakamae A, Ochi M, Deie M et al (2014) Clinical outcomes of second-look arthroscopic evaluation after anterior cruciate ligament augmentation: comparison with single- and double-bundle reconstruction. Bone Joint J 96-b:1325-1332

33. Choi S, Kim MK, Kwon YS, Kang H (2017) Clinical and arthroscopic outcome of single bundle anterior cruciate ligament reconstruction: Comparison of remnant preservation versus conventional technique. Knee 24:1025-1032

34. Xu H, Dong J, Xin D et al (2017) Second-look arthroscopic evaluation and clinical outcomes of anatomic anterior cruciate ligament reconstruction with autograft and hybrid graft: A retrospective study. Med Sci Monit 23:5564-5573

35. Falconiero RP, DiStefano VJ, Cook TM (1998) Revascularization and ligamentization of autogenous anterior cruciate ligament grafts in humans. Arthroscopy 14:197-205

36. Sánchez M, Anitua E, Azofra J et al (2010) Ligamentization of tendon grafts treated with an endogenous preparation rich in growth factors: gross morphology and histology. Arthroscopy 26:470-480

37. Rougraff B, Shelbourne KD, Gerth PK, Warner J (1993) Arthroscopic and histologic analysis of human patellar tendon autografts used for anterior cruciate ligament reconstruction. Am J Sports Med 21:277-284

Ready to submit your research? Choose BMC and benefit from:

- fast, convenient online submission

- thorough peer review by experienced researchers in your field

- rapid publication on acceptance

- support for research data, including large and complex data types

- gold Open Access which fosters wider collaboration and increased citations

- maximum visibility for your research: over $100 \mathrm{M}$ website views per year

At $\mathrm{BMC}$, research is always in progress.

Learn more biomedcentral.com/submissions 